\title{
Triple-negative breast cancer: current management and future options
}

\author{
PierFranco Conte*, Valentina Guarneri \\ Department of Oncology and Hematology, University Hospital, Modena, Italy
}

\section{ARTICLE INFO}

\section{Keywords:}

Metastatic breast cancer

Triple-negative

Chemotherapy

Epothilones

\begin{abstract}
Triple-negative breast cancer is a particularly difficult to treat and biologically aggressive disease with limited treatment options. However, a subset of patients with triple-negative tumours may respond to chemotherapy. Therefore, optimisation of chemotherapy regimens may be key in treating triple-negative breast cancer. Emerging treatment approaches include novel chemotherapeutic agents such as the epothilones. The epothilones are a group of novel microtubule-stabilising agents with demonstrated activity in anthracycline-/taxane-resistant triple-negative breast cancer, and ongoing trials are evaluating the combination of epothilones with targeted agents or inclusion of epothilones in novel combination regimens. Other interesting new treatment options include the PARP inhibitors, which are currently in clinical trials for triple-negative breast cancer.
\end{abstract}

() 2009 Elsevier Ltd. All rights reserved.

\section{Introduction}

Hormone receptor (oestrogen and progesterone) and human epidermal growth factor receptor 2 (HER2) status identify key molecular subtypes of human breast tumours and currently guide choice of therapy.

For patients with hormone-receptor positive metastatic breast cancer (MBC), hormone therapy with tamoxifen, fulvestrant, and aromatase inhibitors (anastrozole, letrozole, exemestane in postmenopausal patients) or ovarian suppression (in premenopausal patients) represent the backbone of treatment, with or without additional chemotherapy. For patients with HER2-positive breast cancer, regardless of any other characteristics, the introduction of anti-HER2 targeted agents has dramatically improved prognosis. ${ }^{1}$

Triple-negative breast cancer lacks overexpression of all three receptors (oestrogen, progesterone and HER2),

${ }^{*}$ Corresponding author. PierFranco Conte.

Tel: +39059 4224538; fax: +390594224429.

E-mail address: conte.pierfranco@unimore.it (P. Conte). and represents $\sim 15 \%$ of all breast cancers. Triple-negative disease is characterised by a high proliferation rate, high risk of early recurrence and a high incidence of visceral and CNS metastases. ${ }^{2,3}$ For this particularly aggressive subset of breast cancer, effective treatment choices are currently limited to chemotherapy; no targeted agents have been developed specifically for triple-negative cancer. For instance, in the neoadjuvant setting, a significant proportion of triple-negative tumours achieve a pathological complete response (pCR) with chemotherapy. It is important to note that the prognosis for triple-negative patients who achieve a pCR with chemotherapy in the neoadjuvant setting is similar to that for other breast cancer phenotypes. ${ }^{4}$ In contrast, patients with triplenegative breast cancer who fail to achieve a pCR have a worse prognosis. ${ }^{5}$ It is therefore important to offer these patients the most effective treatments as early as possible in their disease course, and to identify predictive and prognostic markers to guide treatment choice.

Treatment options for patients in whom disease recurs vary according to key patient- and diseaserelated factors. These include age, site and extent of 
metastases, treatment-free interval, and prior adjuvant chemotherapy. Due to the high risk of relapse and lack of other therapeutic options, the majority of triple-negative breast cancer patients receive adjuvant chemotherapy. This typically includes anthracyclines and/or taxanes. Therefore, in case of relapse, treatment options are further limited by the prior exposure to therapy and the resulting emergent treatment resistance. In recent years, a number of new treatment options have emerged for patients with triple-negative disease. These include a novel group of cytotoxic agents, the epothilones, as well as agents with molecular targets. This article reviews the latest developments for treating patients with triplenegative breast cancer.

\section{Combination chemotherapy in triple-negative disease}

Although combination chemotherapy is routinely used in the adjuvant setting, its use for treating MBC is more controversial. ${ }^{6}$ Clinical studies have shown that combination therapy offers only a limited survival advantage compared with single-agent therapy or sequential monotherapy. ${ }^{7-10}$ An additional consideration for selecting combination therapy is whether the efficacy benefits associated with combining treatment options outweigh the risks of additive drug side effects. Consequently, the disadvantage of increased toxicity and diminished quality of life during treatment have generally been regarded as sufficient to negate the minimal benefit that combination therapy might offer. ${ }^{11}$

Nevertheless, data do suggest that combination therapy may offer improved outcomes in terms of progression-free survival (PFS). For example, a recent study of vinorelbine plus gemcitabine vs vinorelbine alone demonstrated a significantly prolonged PFS for the combination regimen (6.0 vs 4.0 months, hazard ratio [HR] 0.66; 95\% confidence interval [CI] 0.50-0.88; $P=0.0028) .{ }^{12}$ Judicious selection of new combination regimes using drugs with minimally overlapping resistance and toxicity profiles may yield future progress. Moreover, combination chemotherapy - as well as novel combinations of cytotoxic and targeted agents - may still be of particular clinical relevance for patients with aggressive triple-negative disease given their extremely poor prognosis, especially once their disease has metastasised.

Novel agents that overcome the common mechanisms of drug resistance and retain clinically relevant activity in the treatment of metastatic disease are of particular relevance in this subset of breast cancer patients.

The epothilones are a novel class of microtubulestabilising agents that are less susceptible to the cellular mechanisms of resistance associated with other chemotherapeutic drug classes. Among the epothilones,

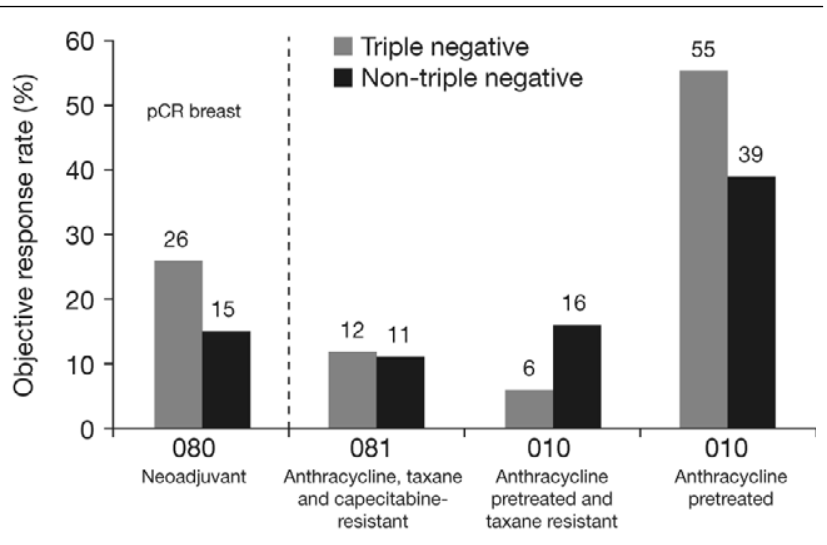

Fig. 1 - Efficacy of ixabepilone monotherapy in triplenegative breast cancer: a subgroup analysis from phase II trials. ${ }^{13}$

ixabepilone (BMS-247550) is the most advanced in clinical development. Indeed, ixabepilone has received approval from the US Food and Drug Administration for the treatment of metastatic or locally advanced breast cancer after failure of an anthracycline and a taxane ${ }^{14}$ and is currently under regulatory review in Europe.

Clinical trials have shown that ixabepilone exhibits potent antitumour effects in all stages of breast cancer, from patients receiving neoadjuvant therapy to those with heavily pre-treated $\mathrm{MBC}^{15-19}$ (see also the paper by Fumoleau in this supplement ${ }^{20}$ ). In a sub-analysis of data from five clinical trials, Pivot and colleagues ${ }^{13}$ found that ixabepilone also demonstrated notable antitumour activity in patients with triple-negative breast cancer. ${ }^{15,16}$ Interestingly, in the neoadjuvant setting, a greater proportion of patients with triple-negative disease achieved a pCR with ixabepilone (breast plus nodes; $\mathrm{pCR}=19 \%$ ) compared with those with hormone receptor positive disease $(\mathrm{pCR}=8 \%)$. This finding was consistent with a report by Liedtke and colleagues for taxanes. ${ }^{21}$ Generally, across the phase II ixabepilone clinical trial program, patients with triple-negative disease achieved overall response rates comparable to those of patients without triple-negative disease (Fig. 1). Median PFS in the phase II trials of ixabepilone given as single-agent therapy ranged from 2.5 to 5.7 months for those without triple-negative disease and from 1.6 to 4.6 months for those with triple-negative disease. ${ }^{13}$

The efficacy and safety of ixabepilone in combination with capecitabine for MBC has also been investigated in two phase III trials (CA163046 and CA163048). In the pivotal study (046), patients were prospectively defined with a strict definition of resistance to previous anthracycline and taxane therapy, ${ }^{19}$ whereas in the second, confirmatory study (048) patients were pretreated or resistant to an anthracycline and a taxane. ${ }^{22}$ In study 046, the overall response rate assessed by independent radiology review was 35\% for patients who received combination therapy compared with $14 \%$ for 


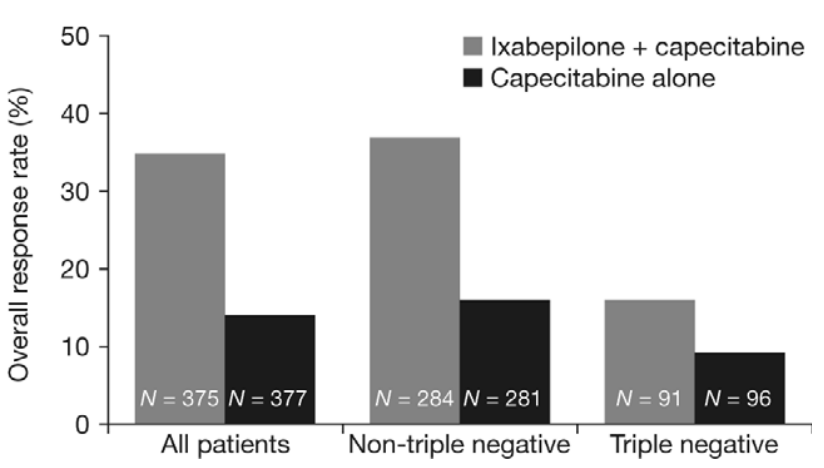

Fig. 2 - Overall response rates among patients treated with ixabepilone + capecitabine or capecitabine alone: overall and subgroup analyses. ${ }^{23}$

those treated with capecitabine alone $(P=0.0001) .{ }^{19}$ Investigator-assessed responses were $42 \%$ and $23 \%$, respectively. Consistent with this, the investigatorassessed overall response rate in study 048 was $42 \%$ in the ixabepilone combination therapy arm and $23 \%$ for capecitabine alone. ${ }^{22}$ Across these two studies, ixabepilone in combination with capecitabine offered an increase in PFS of approximately $40 \%$ compared with capecitabine alone. When outcomes for the subgroup of patients with triple-negative disease who took part in study 046 were evaluated, the results were consistent with the overall findings. PFS was prolonged in patients with triple-negative disease from 2.1 months among those who received capecitabine alone to 4.1 months among those treated with ixabepilone plus capecitabine, indicating a $32 \%$ reduction in the risk of progressive disease compared with capecitabine alone. ${ }^{23}$ Moreover, there was a three-fold increase in the overall response rate in patients with triple-negative disease, from 9\% among those who received capecitabine alone to $27 \%$ among those treated with the combination regimen (Fig. 2).

\section{Novel molecular targets in triple-negative disease}

Targeted agents have also been investigated in triplenegative patient populations. For example, clinical trials of bevacizumab in combination with either paclitaxel or docetaxel as first-line therapy for patients with metastatic or locally advanced breast cancer demonstrated that combination treatment significantly increased both the objective response rate and the median PFS compared with monotherapy. ${ }^{24,25}$ Miller and coworkers (2007) reported a significant improvement in the overall response rate with paclitaxel plus bevacizumab as first-line therapy for MBC (36.9\% vs $21.2 \%$ for paclitaxel alone; $P=0.0001)$. In this study, patients also experienced a statistically significant improvement in PFS with combined paclitaxel plus bevacizumab compared with paclitaxel alone (11.8 vs 5.9 months, $P<0.001$ ) but no significant improvement in overall survival (26.2 vs 25.2 months). In particular, the addition of bevacizumab to paclitaxel resulted in a similar PFS benefit in both the triple-negative and hormone receptor positive groups (HR 0.53 and 0.54, respectively). Similarly, significant improvements in both overall response rate and PFS have been reported for the combination of docetaxel and bevacizumab compared with docetaxel alone as first-line therapy for locally advanced or metastatic breast cancer. ${ }^{25}$ In this case, there was a trend towards improved 1-year survival with combination therapy. Again, the subgroup analysis has highlighted a similar benefit for patients with triplenegative breast cancer.

A variety of alternative rational molecular targets for the treatment of patients whose disease is HER2negative, including those with triple-negative disease, has emerged in recent years. ${ }^{26}$

Triple-negative tumours have many histological similarities to the subgroup of breast tumours that carry a mutation in the Breast Cancer 1 (BRCA-1) gene. The BRCA1 gene product acts as a tumour suppressor by maintaining genomic integrity via DNA damage repair, ubiquitination and transcriptional regulation. ${ }^{27}$ Loss of functional BRCA1 results in the accumulation of genetic mutations, genomic instability and a predisposition towards oncogenic transformation. Such cells depend on base excision repair (BER) mechanisms to rectify endogenous DNA damage and poly(ADP-ribose) polymerase (PARP) enzyme is a critical component of BER. PARP inhibition has been shown to be effective in killing BRCA-1-mutant cell lines, including breast cancer cell lines, ${ }^{28}$ and such inhibitors may sensitise cancer cells to DNA-damaging agents. ${ }^{29}$ PARP inhibitors including AZD2281 in combination with paclitaxel (study code: NCT00707707) or as monotherapy (study code: NCT00679783), and BSI-201 in combination with carboplatin plus gemcitabine (study code: NCT00540358), are currently in early clinical development and their potential in the treatment of triple-negative MBC awaits further clinical evaluation.

Other potential molecular targets for patients with triple-negative disease are also under investigation. For example, between $50 \%$ and $70 \%$ of triple-negative tumours have been shown to overexpress the epidermal growth factor receptor (EGFR), around 31\% overexpress the c-Kit receptor which on binding of stem cell factor initiates a signalling cascade that is thought to promote cell survival, proliferation and migration, and overexpression of src (a tyrosine kinase involved in growth cellular signal propagation) has also been reported in triple-negative breast tumours. ${ }^{26}$ These observations provide a rational basis for the evaluation of EGFRtargeted agents such as cetuximab and erlotinib, c-Kit 
targeted agents such as imatinib and src-targeted agents such as dasatinib in the treatment of triple-negative breast cancer. Preliminary data with the EGFR inhibitor cetuximab suggest that combination therapy with carboplatin may be effective in patients with triple-negative disease. ${ }^{30}$ Among a population of patients with stage IV, triple-negative breast cancer, most of whom had received prior treatment for metastatic disease, the response rate for patients treated with cetuximab alone was low with only $6 \%$ of patients responding. This improved to $17 \%$ when carboplatin was added on disease progression, a rate that was comparable with a response rate of $17 \%$ observed for patients randomised to cetuximab plus carboplatin throughout. However, while the improved response rates appear to favour combination therapy, the differences between treatment arms failed to reach statistical significance. In a subset analysis investigating the effects of adding cetuximab to irinotecan and carboplatin in 72 patients with triple-negative breast cancer, cetuximab increased the objective response rate from $30 \%$ to $49 \% .{ }^{31}$ However, the improvement in response rates was not associated with any improvement in PFS (irinotecan plus carboplatin 5.1 months vs 4.7 months for irinotecan plus carboplatin plus cetuximab).

Given these results, a phase II study is now underway to examine the efficacy of ixabepilone in combination with cetuximab in patients with triple-negative locally advanced or metastatic breast cancer (study code: NCT00633464). Patients who have not received prior chemotherapy in the metastatic setting with confirmed triple-negative disease will be randomised to ixabepilone only or ixabepilone in combination with cetuximab, and will be followed until disease progression or withdrawal for unacceptable toxicity. The primary endpoint of the study will be the overall response rate, with PFS, time to response and duration of response as secondary study endpoints. Final results of this study are expected in 2011.

\section{Conclusions}

Triple-negative MBC represents a considerable clinical challenge. Although triple-negative tumours are more likely to undergo early relapse and develop visceral metastases, patients with this poor prognosis subtype can achieve substantial benefits with chemotherapy. However, treatment choices for patients with triplenegative MBC are limited by often heavy pre-treatment with the most effective chemotherapeutic agents. Accordingly, there is a need for new agents that are effective in triple-negative disease which can overcome the resistance mechanisms that preclude the sustained efficacy of the anthracyclines and taxanes in the metastatic disease setting. The epothilones, notably ixabepilone, the most developmentally advanced agent in this group, are one such new group of cytotoxic agents that retain activity in anthracycline- and taxaneresistant breast cancer, and are emerging as a valuable alternative for patients with $\mathrm{MBC}$ including triplenegative disease.

The growing appreciation of the use of combination chemotherapy with newer cytotoxics, with improved efficacy and/or a more tolerable side-effect burden compared with older agents and regimens, has opened new potential treatment options particularly for those with triple-negative disease. Such regimens are gaining increasing acceptance in the management of late-stage disease. Phase II studies have demonstrated the activity of ixabepilone in the treatment of triple-negative disease from early stage through to heavily pre-treated $M B C$, and data from phase III studies support combination chemotherapy with ixabepilone and capecitabine with indications of both improved response and prolonged progression-free intervals.

Novel molecular targets in triple-negative breast cancer are also emerging and providing a rationale for the evaluation of a number of novel combination regimens in triple-negative breast cancer. Again, there is early evidence of improved response rates and it is hoped that ongoing studies with regimens such as that of ixabepilone in combination with cetuximab may prove effective in extending progression-free periods for patients with triple-negative MBC.

Patients with metastatic triple-negative breast cancer face a bleak prognosis. However, with the emergence of novel chemotherapeutics, evidence of efficacy and tolerability of novel combination regimens and the incorporation of novel targeted agents into chemotherapeutic regimens, there remains hope for improved outcomes for this vulnerable and under-served group of patients.

Acknowledgements: Medical writing and editorial assistance, provided by Gardiner-Caldwell U.S., was supported by Bristol-Myers Squibb.

Competing interests: Dr Conte has acted as consultant and speaker for Bristol-Myers Squibb. Dr Guarneri indicated no potential conflicts of interest.

\section{REFERENCES}

1. Dawood S, Kristine B, Hortobagyi, GN, Giordano SH. Prognosis of women with stage IV breast cancer by HER2 status and trastuzumab treatment: An institutional based review. J Clin Oncol 2008;26:(15S): abstract 1018.

2. Bauer KR, Brown M, Cress RD, Parise CA, Caggiano V. Descriptive analysis of estrogen receptor (ER)-negative, progesterone receptor (PR)-negative, and HER2-

negative invasive breast cancer, the so-called triplenegative phenotype. A population-based study from the California Cancer Registry. Cancer 2007;109:1721-8. 
3. Heitz F, Harter P, Traut A, Lueck HJ, Beutel B, du Bois A. Cerebral metastases (CM) in breast cancer (BC) with focus on triple-negative tumors. J Clin Oncol 2008;26(15S): abstract 1010.

4. Rouzier R, Pusztai L, Delaloge S, et al. Nomograms to predict pathologic complete response and metastasisfree survival after preoperative chemotherapy for breast cancer. J Clin Oncol 2005;23:8331-9.

5. Carey LA, Dees EC, Sawyer L, et al. The triple-negative paradox: primary tumor chemosensitivity of breast cancer subtypes. Clin Cancer Res 2007;13:2329-34.

6. Miles D, von Minckwitz G, Seidman AD. Combination versus sequential single-agent therapy in metastatic breast cancer. Oncologist 2002;7(Suppl 6):13-9.

7. Conte PF, Guarneri V, Bruzzi P, et al. Concomitant versus sequential administration of epirubicin and paclitaxel as first-line therapy in metastatic breast carcinoma: results for the Gruppo Oncologico Nord Ovest randomized trial. Cancer 2004;101:704-12.

8. Fossati R, Confalonieri C, Torri V, et al. Cytotoxic and hormonal treatment for metastatic breast cancer: a systematic review of published randomized trials involving 31,510 women. J Clin Oncol 1998;16:3439-60.

9. Sledge GW, Neuberg D, Ingle J, et al. Phase III trial of doxorubicin, paclitaxel and the combination of doxorubicin and paclitaxel as front-line chemotherapy for metastatic breast cancer: an intergroup trial (E1193). J Clin Oncol 2003;21:588-92.

10. Joensuu H, Holli $\mathrm{K}$, Heikkinen $\mathrm{M}$, et al. Combination chemotherapy versus single-agent therapy as firstand second-line treatment in metastatic breast cancer: a prospective randomized trial. J Clin Oncol 1998;16(12):3720-30.

11. Alba E, Martín M, Ramos M, et al. Multicenter randomized trial comparing sequential with concomitant administration of doxorubicin and docetaxel as first-line treatment of metastatic breast cancer: a Spanish Breast Cancer Research Group (GEICAM-9903) phase III study. J Clin Oncol 2004;22:2587-93.

12. Martín M, Ruiz A, Muñoz M, et al. Gemcitabine plus vinorelbine versus vinorelbine monotherapy in patients with metastatic breast cancer previously treated with anthracyclines and taxanes: final results of the phase III Spanish Breast Cancer Research Group (GEICAM) trial. Lancet Oncol 2007;8:219-25.

13. Pivot $X$, Llombart-Cussac A, Martin $M$, et al. Clinical activity of the novel epothilone $\mathrm{B}$ analog, ixabepilone, in triple negative breast cancer (BC) patients. EJC Suppl 2008;6(7):134. Abstract 290.

14. IXEMPRA TM Kit (Ixabepilone) for injection [package insert]. Princeton, NJ: Bristol-Myers Squibb Company; 2007.

15. Baselga J, Gianni L, Llombart A, et al. Predicting response to ixabepilone: genomic study in patients receiving single agent ixabepilone as neoadjuvant treatment for breast cancer. Breast Cancer Res Treat 2005;94: abstract 305.

16. Perez EA, Lerzo G, Pivot X, et al. Efficacy and safety of ixabepilone (BMS0247550) in a phase II study of patients with advanced breast cancer resistant to an anthracycline, a taxane, and capecitabine. J Clin Oncol 2007;25:3407-14.
17. Roché $\mathrm{H}$, Yelle L, Cognetti F, et al. Phase II clinical trial of ixabepilone (BMS-247550), an epothilone B analog, as first-line therapy in patients with metastatic breast cancer previously treated with anthracycline chemotherapy. J Clin Oncol 2007;25:3415-20.

18. Thomas E, Tabernero J, Fornier M, et al. Phase II clinical trial of ixabepilone (BMS-247550), an epothilone B analog, in patients with taxane-resistant metastatic breast cancer. J Clin Oncol 2007;25:3399-406.

19. Thomas ES, Gomez HL, Li RK, et al. Ixabepilone plus capecitabine for metastatic breast cancer progressing after anthracycine and taxane treatment. J Clin Oncol 2007;25:5210-7.

20. Fumoleau P. Treatment beyond taxanes, emerging new cytotoxic agents. EJC Suppl 2009;7(1):8-13.

21. Liedtke C, Mazouni C, Hess KR, et al. Response to neoadjuvant therapy and long-term survival in patients with triple-negative breast cancer. J Clin Oncol 2008 Mar 10;26(8):1275-81.

22. Hortobagyi GN, Perez E, Vrdoljak E, et al. Analysis of overall survival (OS) among patients (pts) with metastatic breast cancer $(\mathrm{MBC})$ receiving either ixabepilone (I) plus capecitabine (C) or C alone: results from two randomized phase III trials. ASCO Breast Cancer Symposium 2008: abstract 186.

23. Rugo H, Thomas ES, Lee RK, et al. Combination therapy with ixabepilone plus capecitabine is effective in ER/PR/ HER2-negative breast cancer resistant to anthracyclines and taxanes. Breast Cancer Res Treat 2007;106(S70): abstract 6069.

24. Miller K, Wang M, Gralow J, et al. Paclitaxel plus bevacizumab versus paclitaxel alone for metastatic breast cancer. N Engl J Med 2007;357:2666-76.

25. Miles D, Chan A, Romieu G, et al. Randomized, double-blind, placebo-controlled, phase III study of bevacizumab with docetaxel or docetaxel with placebo as first-line therapy for patients with locally recurrent or metastatic breast cancer (mBC): AVADO. J Clin Oncol 2008;26(15S): abstract LBA1011.

26. Cleator S, Heller W, Coombes RC. Triple-negative breast cancer: therapeutic options. Lancet Oncol 2007;8(3):235-44.

27. Starita LM, Parvin JD. The multiple nuclear functions of BRCA1: transcription, ubiquitination and DNA repair. Curr Opin Cell Biol 2003;15:345-50.

28. Drew Y, Calvert $H$. The potential of PARP inhibitors in genetic breast and ovarian cancers. Ann NY Acad Sci 2008;1138:136-45.

29. Muñoz-Gámez JA, Martín-Oliva D, Aguilar-Quesada R, et al. PARP inhibition sensitizes p53-deficient breast cancer cells to doxorubicin-induced apoptosis. Biochem J 2005;386:119-25.

30. Carey LA, Rugo HS, Marcom PK, et al. TBCRC 001: EGFR inhibition with cetuximab added to carboplatin in metastatic triple-negative (basal-like) breast cancer. J Clin Oncol 2008;26(15S): abstract 1009.

31. O'Shaughnessy J, Weckstein D, Vukelja SJ, et al. Randomized phase II study of weekly irinotecan/ carboplatin with or without cetuximab in patients with metastatic breast cancer. Breast Cancer Res Treat 2007;106: abstract 308. 